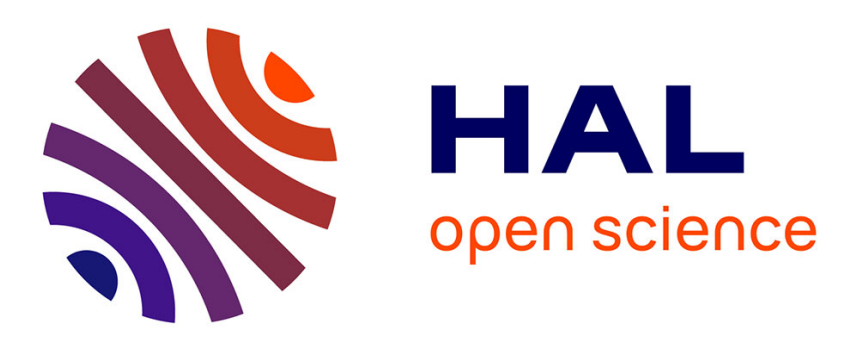

\title{
Note complémentaire sur l'origine de la dédicace d'un nauclerus découverte à Fos-sur-Mer
}

\author{
Frédéric Marty, Cyril Courrier, Philippe Bromblet
}

\section{To cite this version:}

Frédéric Marty, Cyril Courrier, Philippe Bromblet. Note complémentaire sur l'origine de la dédicace d'un nauclerus découverte à Fos-sur-Mer. Revue archéologique de Narbonnaise, 2018, 49, pp.367-372. 10.3406/ran.2016.1942 . halshs-03407008

\section{HAL Id: halshs-03407008 \\ https://shs.hal.science/halshs-03407008}

Submitted on 6 Nov 2021

HAL is a multi-disciplinary open access archive for the deposit and dissemination of scientific research documents, whether they are published or not. The documents may come from teaching and research institutions in France or abroad, or from public or private research centers.
L'archive ouverte pluridisciplinaire HAL, est destinée au dépôt et à la diffusion de documents scientifiques de niveau recherche, publiés ou non, émanant des établissements d'enseignement et de recherche français ou étrangers, des laboratoires publics ou privés. 


\title{
Note complémentaire sur l'origine de la dédicace d'un nauclerus découverte à Fos-sur-Mer
}

\author{
- Frédéric Marty \\ - Cyril Courrier \\ - Philippe Bromblet
}

Résumé:

La mise au jour d'une série de clichés photographiques dans les archives de Charles Massard, ancien secrétaire des "Amis du Vieux Fos», permet de préciser l'origine d'un autel (ou d'une base de statue) inscrit, récemment publié dans la RAN (Courrier 2015). Par ailleurs, les résultats de l'étude d'une lame mince prélevée en septembre 2015 sur le monument donnent des indices quant à la provenance du matériau.

\section{Mots-clés :}

Narbonnaise, Arles, Fos-sur-Mer, Charles Massard, archives, port, dédicace, Genius, nauclerus, armateurs, negotiantes, négociants, commerce.

\section{Abstract:}

The discovery of a series of photographs among Charles Massard's archives, former secretary of the "Amis du Vieux Fos», allows us to establish beyond doubt the origin of an inscribed altar or a pedestal recently published in the RAN journal (Courrier 2015). Furthermore, the analysis of a sample taken in September 2015 also provides indications about the stone's geological provenance.

Keywords:

Narbonnaise, Arles, Fos-sur-Mer, Charles Massard, archives, port, dedication, Genius, nauclerus, shippers, negotiantes, traders, merchants.

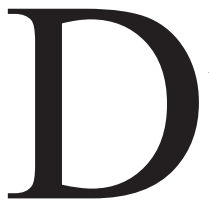

ans le numéro 48 de la Revue Archéologique de Narbonnaise, Cyril Courrier publiait un autel ou une base de statue (fig. 1) portant la dédicace d'un nauclerus à une divinité considérée comme le Génie, ou à une divinité et au Génie, d'un groupe de negotiantes subaediani (Courrier 2015). Cet objet, qui provient sans conteste du port antique de Fos-sur-Mer, posait toutefois un problème de localisation dans la mesure où certains documents d'archives en situaient la découverte à l'emplacement du gisement sous-marin d'autels et de stèles funéraires de l'anse Saint-Gervais, tandis que d'autres, qui avaient eu la préférence de l'auteur, suggéraient qu'il pouvait provenir des travaux de construction du port de plaisance. Une recherche dans les archives de Charles Massard ${ }^{1}$, menée a posteriori pour une autre enquête, permet à présent de lever définitivement le doute au profit de la seconde hypothèse $(\S 1)$. Par ailleurs, les résultats de l'étude récente d'une lame mince prélevée en septembre 2015 donnent des indices quant à la provenance du matériau $(\$ 2)$. 
Figure 1

Relevé du monument offert par le nauclère (DAO Fr. Marty).

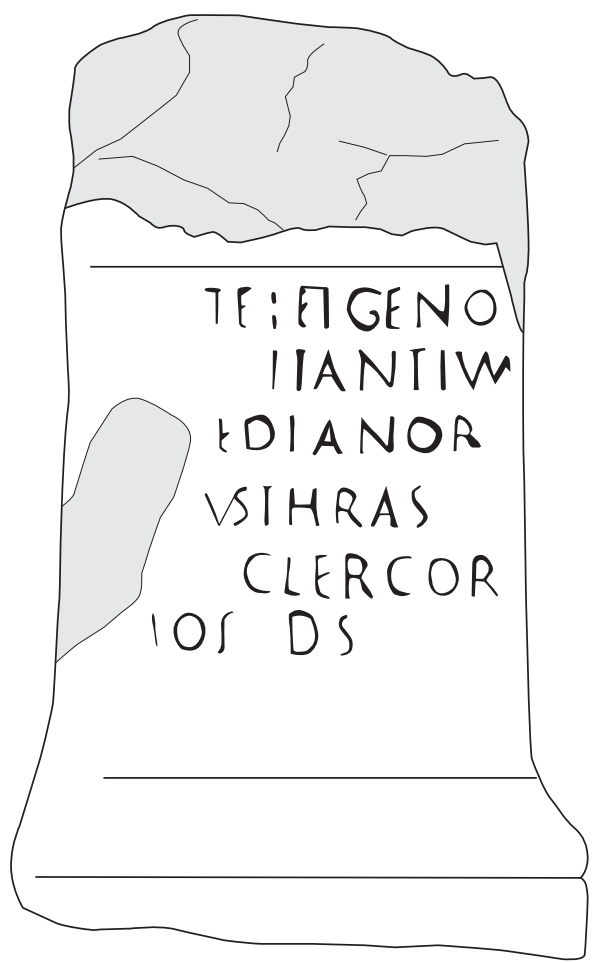

0 $50 \mathrm{~cm}$

\section{LE MONUMENT DU NAUCLÈRE dans les archives de Charles Massard}

Charles Massard (1923-2000), radio-électricien de métier, est venu à l'archéologie dans les années 1960 par la lecture d'un ouvrage de vulgarisation traitant de l'archéologie provençale (Clébert 1966; Massard 1993). Il s'est alors consacré, en amateur, à la fouille du site grec de la Roque d'Odor voué à être détruit par les travaux de construction du port autonome de Marseille (Marty 2017). Auparavant, Fossur-Mer était son lieu de vacances. C'est là qu'il a assisté, en 1960-1961, aux débuts de la plongée autonome et remonté, comme plusieurs de ses amis, des amphores de l'anse Saint-Gervais. En 1967, naissent les «Amis du Vieux Fos» dont il devient le secrétaire dès la création. L'association est principalement composée de plongeurs qui alimentent, avec le produit de leurs ramassages sous-marins, un musée aménagé dans la chapelle Notre-Dame-de-la-Mer et inauguré le 3 juin 1978. Pour une discipline encore très jeune et mobilisant presque exclusivement des amateurs, c'est l'époque de la recherche du bel objet. Charles Massard est toutefois conscient de l'intérêt scientifique de la collection. C'est pourquoi il entreprend de dessiner et de mesurer les amphores conservées dans la chapelle en vue de leur future étude. Par ailleurs, afin de sensibiliser les autres membres de l'association à la démarche archéologique, il organise, avec le Syndicat d'Initiative, des conférences présentées par Bernard Liou, professeur à l'Université de Provence et directeur de la DRASM.

En 1978, démarrent les travaux de construction du port de plaisance au sud de la pointe de Saint-Gervais. À cette époque, ce que l'on appelle alors «l'archéologie de sauvetage» n'a pas encore de cadre légal. Aussi les associations culturelles locales telles que les «Amis du Vieux Fos» jouent-elles un rôle important dans la sauvegarde du patrimoine, malgré des conditions d'activité contraignantes. Pour intervenir, l'association bénéficie cependant d'une autorisation pour dix de ses membres, nommément désignés, délivrée par la DRASM le 22 novembre 1978, sous l'autorité de Bernard Liou. Le document, conservé dans les archives des Amis du Vieux-Fos, stipule que les fouilleurs, dont fait partie Charles Massard, sont « autorisés à collecter dans les déblais de dragage [...] le matériel archéologique qui peut s’y trouver». «Ces recherches ne peuvent se faire qu'aux heures d'arrêt du chantier» sous la seule responsabilité des participants. Ce sera l'un des moments forts de l'activité archéologique de l'association. Un inventaire daté du 20 avril 1979, joint à la déclaration de découverte, montre la diversité des artéfacts retrouvés et leur importance quantitative, souvent estimée par des chiffres arrondis faute de pouvoir tout compter précisément: plus de 2000 fragments de vaisselle en céramique, plus de 200 fragments d'amphores, plus de 100 céramiques autres, 200 fragments de verre, 8 objets en liège ou en bois, 8 objets en os, près de 200 objets en métal, 596 monnaies et 44 objets en pierre (Courrier 2015, 15, fig. 9). Parmi ces derniers, sont listées trois stèles, dont une avec inscription, que Cyril Courrier avait proposé d'identifier au monument du nauclère (Courrier 2015, 13).

Durant l'opération, Charles Massard prend une série continue de huit clichés, numérotés de 6 à 13, dont Frédéric Marty a retrouvé la planche contact (fig. 2a-2e). Les clichés 6 à 7 et 10 à 13, pris de différents points de vue durant la même journée, donnent une vision globale du chantier à l'endroit qui deviendra le port de plaisance. Insérés dans cette série, les clichés 8 et 9 montrent le monument offert par le nauclère aux négociants du port antique de Fos, avec son inscription bien reconnaissable et dans un état identique à l'actuel, couché sur le fond d'un petit bateau de pêche que l'on aperçoit à droite de la drague, sur le cliché 7. Pendant ce temps, à terre, quatre personnes sont visiblement en train d'inspecter les tas de déblais. L'examen des différentes photographies permet de localiser assez précisément l'emplacement de la drague. Elle se situe, sans ambiguïté, au niveau des bureaux de la capitainerie construits par 

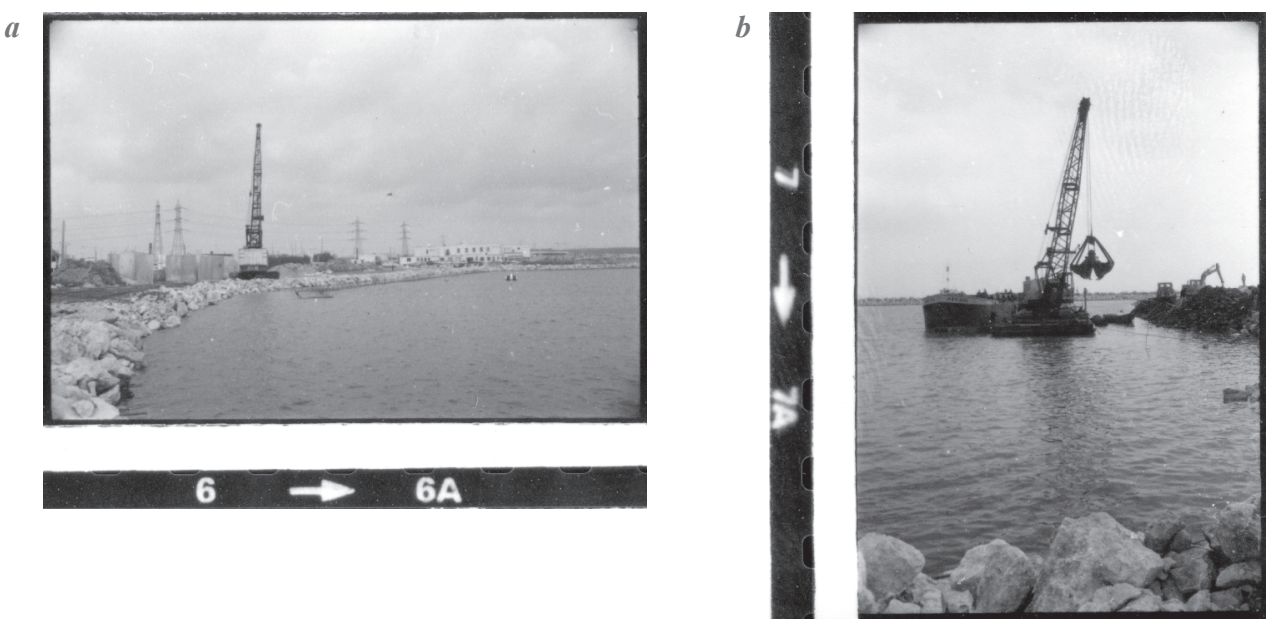

- Figure 2

Travaux d'aménagement du port de plaisance de Saint-Gervais et découverte du monument offert par le nauclère (clichés Ch. Massard).
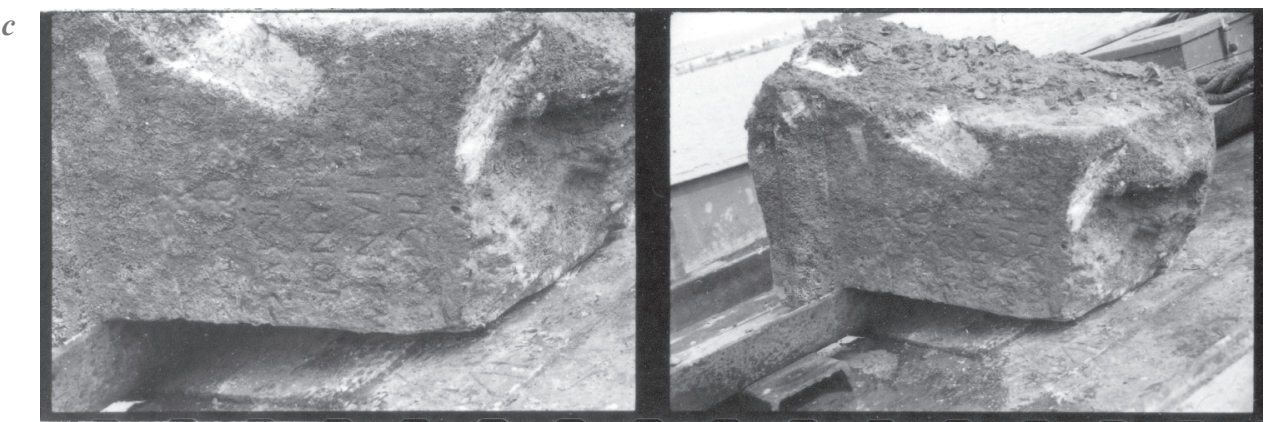

$8 \Rightarrow 8 A$

$9 \Rightarrow 9$
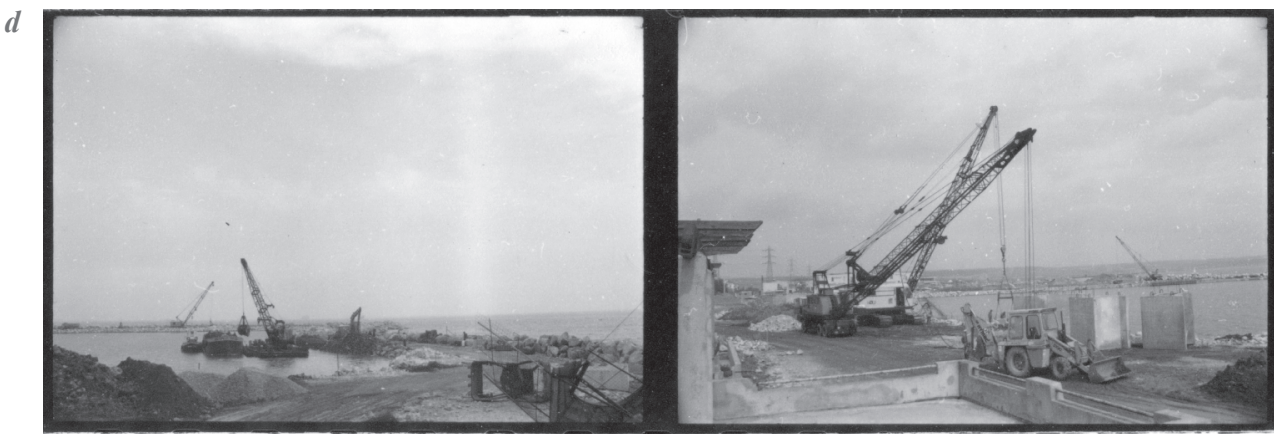

10

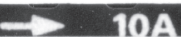

11

$11 \mathrm{~A}$

$e$

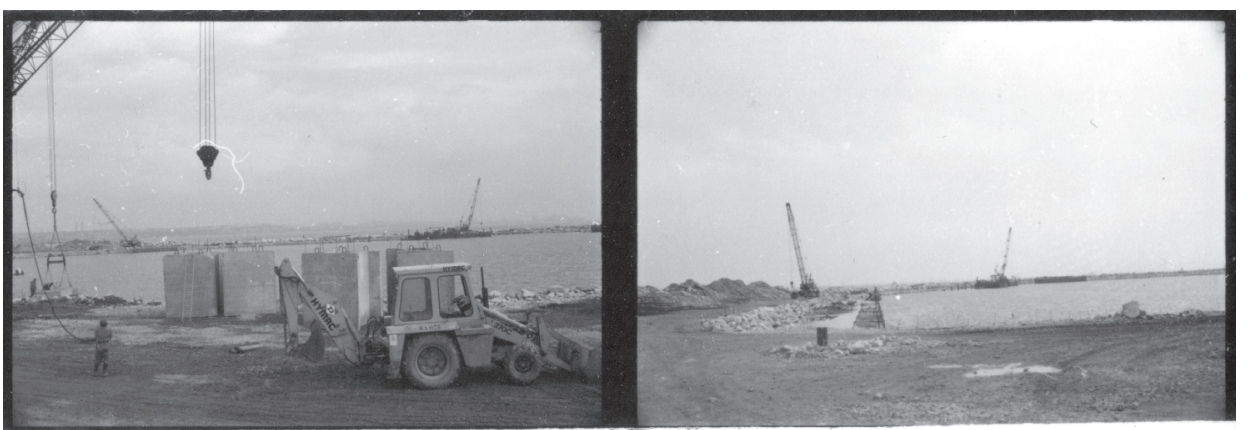

12

$\Rightarrow \quad 124$ 
Figure 3

Localisation du point de découverte du monument offert par le nauclère, au sud de la pointe de Saint-Gervais (DAO Fr. Marty).

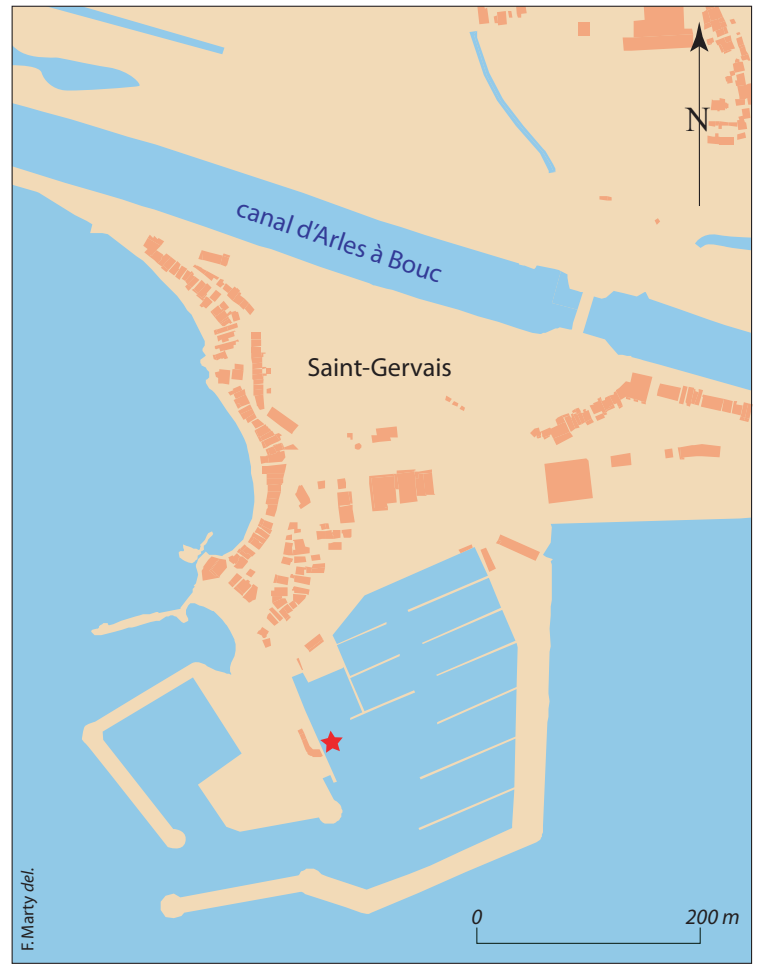

la suite (fig. 3). C'est ainsi que nous obtenons le lieu approximatif de découverte du monument. Son réel contexte archéologique (espace d'exposition, lieu de mise au rebut, réemploi dans une construction...), quant à lui, semble avoir été définitivement effacé par les travaux portuaires. Toutefois, comme Cyril Courrier l'avait suggéré, les clichés pris par Charles Massard ne sont pas sans étayer l'idée que l'objet puisse correspondre à la «stèle» figurée immédiatement au sud de l'atelier de potier sur le plan réalisé en 2009 par Frédéric Marty (Courrier 2015, 13 et 16, fig. 10) sur la base de deux schémas plus anciens dessinés, pour l'un dans les années 1990 (il est anonyme), pour l'autre au début des années 2000 (2004 ou 2005) par Robert Leffy à partir de ses croquis et de mémoire, lesquels représentent, en effet, ce qui semble être une stèle comme si elle comportait une inscription. Rappelons qu'elle est située, sur ce dernier plan, à côté d'un bloc de pierre mortaisé, à quelques mètres (la distance est nettement plus importance sur le plan le plus ancien) d'un bâtiment en pierre représenté par deux murs reliés à angle droit et environnés d'objets domestiques divers, tels qu'un manche de couteau en os en forme de suidé, deux lampes à huile et de la sigillée. Ce bâtiment est lui-même implanté immédiatement au sud-est d'un atelier de potiers caractérisé, notamment, par la présence d'un fond de dolium rempli de surcuits de lampes à huile et de gobelets à paroi fine. Il demeure difficile d'en dire beaucoup plus sur le contexte archéologique, sinon que les vestiges, découverts près du rivage, sont à l'origine terrestres. On peut a priori exclure les restes d'une épave dans la mesure où, dans ce secteur, on ne connaît que le navire Saint-Gervais $2 \mathrm{du} \mathrm{VII}^{\mathrm{e}} \mathrm{s}$.

\section{UNE PROVENANCE GÉOLOGIQUE LOCALE?}

Par ailleurs, afin de tenter de déterminer l'origine de la roche employée, Philippe Bromblet a réalisé l'analyse pétrographique d'une lame mince (fig. 4a-4d). L'examen macroscopique révèle un calcaire blanc

\section{Figure 4}

Photos de la lame mince grossie 25 fois au microscope en lumière polarisée $(L P)$ et en lumière polarisée analysée (LPA) (clichés Ph. Bromblet).
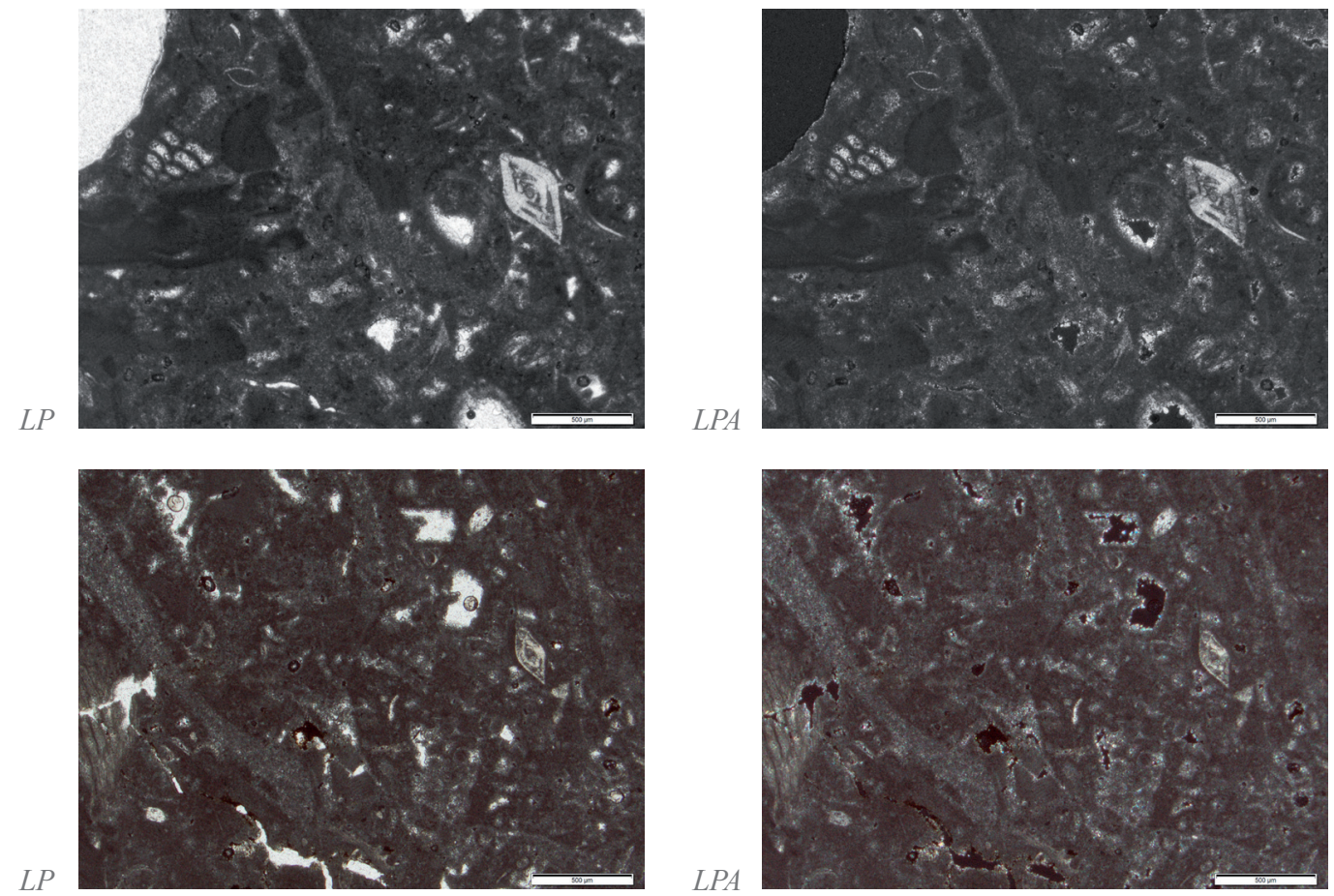

$L P A$

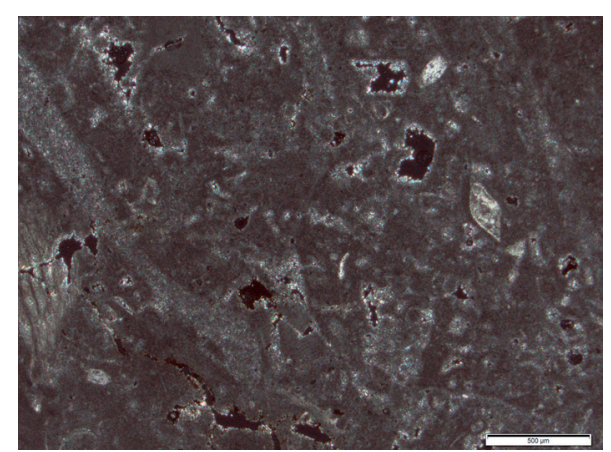


à grain fin, compact et riche en bioclastes. C'est une biocalcarénite blanche. Au microscope, on observe un ciment micritique abondant, de nombreux bioclastes non jointifs à jointifs (texture de type wackestone à packstone) avec des microfossiles (foraminifères de type nummulite) et des fragments bioclastiques divers: algues rouges, plaques d'échinoderme, ostracodes, lamellibranches, bryozoaires, bioturbations. Il s'agit vraisemblablement d'un calcaire d'âge tertiaire. La porosité se présente sous forme de vacuoles, généralement de taille inframillimétrique (max. $2 \mathrm{~mm}$ ), dont les parois sont parfois bordées de calcite microsparitique tardive.

Dans la notice géologique de la carte BRGM n 1019 (Istres), l'étage Burdigalien noté mlb (Miocène) est décrit comme une calcarénite blanche avec, juste au-dessus d'un conglomérat grossier basal, quatre à cinq mètres de calcaire biodétritique blanc à algues, foraminifères, bryozoaires, balanes, oursins... Cette formation, qui affleure en bordure de mer (pointe de Saint-Gervais, Port-de-Bouc...) et sur les falaises blanches à l'arrière-plan, correspond au faciès de cet échantillon. Un contrôle de terrain, comprenant la collecte et l'étude d'échantillons prélevés sur les affleurements, permettrait de confirmer cette éventuelle provenance locale. Les quelques échantillons prélevés jusqu'ici localement (Fos-surMer, Ponteau...) diffèrent cependant visuellement de la pierre du monument du nauclère, beaucoup plus fine et compacte. Par conséquent, son origine géographique exacte reste à déterminer. Dans tous les cas, si la pierre devait être régionale, elle aurait été soigneusement sélectionnée pour ses qualités esthétiques donnant au spectateur, à première vue, l'illusion d'un marbre blanc.

\section{Conclusion}

Quoique la mise au jour des clichés pris par Charles Massard lors des travaux de dragage du port de plaisance ne permette pas d'approfondir l'analyse sur le plan historique, elle confirme malgré tout la lecture épigraphique qui avait été faite de l'inscription en 2015. Surtout, elle donne un lieu de découverte, même approximatif, à ce monument que l'on peut définitivement déconnecter de la «nécropole» de l'anse Saint-Gervais. Au-delà, elle rappelle le rôle des associations et, en leur sein, des figures de l'archéologie locale, de même qu'elle éclaire l'importance de leur patient travail de collecte et d'enregistrement d'informations que l'historien peut, quarante ans plus tard, reprendre avec profit. Dans le cas présent, elle jette une lumière essentielle sur la genèse d'une discipline, dans des conditions légales et matérielles peu évidentes, et souligne le sens du patrimoine, local et antique, qui s'est enraciné dans ces années-là.

\section{Note de commentaire}

1. Archives conservées par le Pôle Intercommunal du Patrimoine Culturel à Fossur-Mer. 


\section{Références bibliographiques}

Clébert 1966: CLÉBERT (J.-P.) - Provence antique. Paris, Robert Laffont, 1966, 281 p.

Courrier 2015: COURRIER (C.) - Une inscription inédite de Fos-sur-Mer: la (vraisemblable) dédicace d'un nauclerus à la divinité tutélaire et au Génie de negotiantes subaediani. RAN, 48, 2015, 9-30.
Marty 2017: MARTY (Fr.) - L'installation littorale grecque de la Roque d'Odor à Fos-sur-Mer (Bouches-du-Rhône). Archaeonautica, 19, 2017, 89-121.

Massard 1993: MASSARD (Ch.) - Ce que je crois être ma contribution à l'archéologie de Fos-sur$\mathrm{Mer}$, tapuscrit, archives du Pôle Intercommunal du Patrimoine Culturel du Territoire Istres OuestProvence, Fos-sur-Mer, v. 1993, 2 p. 